Kragujevac Journal of Mathematics

Volume 45(4) (2021), Pages 521-529.

\title{
PERFECT NILPOTENT GRAPHS
}

\author{
M. J. NIKMEHR ${ }^{1}$ AND A. AZADI ${ }^{1}$
}

\begin{abstract}
Let $R$ be a commutative ring with identity. The nilpotent graph of $R$, denoted by $\Gamma_{N}(R)$, is a graph with vertex set $Z_{N}(R)^{*}$, and two vertices $x$ and $y$ are adjacent if and only if $x y$ is nilpotent, where $Z_{N}(R)=\{x \in R \mid x y$ is nilpotent, for some $\left.y \in R^{*}\right\}$. A perfect graph is a graph in which the chromatic number of every induced subgraph equals the size of the largest clique of that subgraph. In this paper, we characterize all rings whose $\Gamma_{N}(R)$ is perfect. In addition, it is shown that for a ring $R$, if $R$ is Artinian, then $\omega\left(\Gamma_{N}(R)\right)=\chi\left(\Gamma_{N}(R)\right)=\left|\operatorname{Nil}(R)^{*}\right|+|\operatorname{Max}(R)|$.
\end{abstract}

\section{INTRODUCTION}

The theory of graphs associated with rings was started by Beck [4] in 1981 and has grown a lot since then. Anderson and Livingston [2] modified Beck's definition and introduced the notion of zero-divisor graph. Surely, this is the most important graph associated with a ring and not only zero-divisor graphs but also various generalizations of it have attracted many researchers, see for instance $[9,11]$ and $[10]$. The zero-divisor graph of a ring $\mathrm{R}$, denoted by $\Gamma(R)$, is a graph with the vertex set $Z(R)^{*}$ and two distinct vertices $x$ and $y$ are joined by an edge if and only if $x y=0$, where $Z(R)$ is set of zero-divisors of $R$. In [6], Chen defined a kind of graph structure of rings. He let all the elements of ring $R$ be the vertices of the graph and two vertices $x$ and $y$ are adjacent if and only if $x y$ is nilpotent. However, in 2010, Li and $\mathrm{Li}$ [10] modified and studied the nilpotent graph $\Gamma_{N}(R)$ of $R$ is a graph with vertex set $Z_{N}(R)^{*}$, and two vertices $x$ and $y$ are adjacent if and only if $x y$ is nilpotent, where $Z_{N}(R)=\left\{x \in R \mid x y\right.$ is nilpotent, for some $\left.y \in R^{*}\right\}$. Note that the usual zero-divisor graph $\Gamma(R)$ is a subgraph of the graph $\Gamma_{N}(R)$. B. Smith determine all values of $n$ for which zero-divisor graph of $\mathbb{Z}_{n}$ is perfect [13]. Also, Patil et al. [12] characterize

Key words and phrases. Weakly perfect graph, perfect graph, chromatic number, clique number. 2010 Mathematics Subject Classification. Primary: 05c15. Secondary: 05C17, 05C15, 05C25.

DOI $10.46793 / \mathrm{KgJMat} 2104.521 \mathrm{~N}$

Received: September 09, 2018.

Accepted: March 01, 2019. 
various algebraic and order structures whose zero-divisor graphs are perfect graph. Therefore, this paper is devoted to study the perfect of a super graph of zero-divisor graphs. First let us recall some necessary notation and terminology from ring theory and graph theory.

Throughout this paper, all rings are assumed to be commutative with identity. We denote by $Z(R), \mathrm{U}(R), \operatorname{Max}(R)$ and $\mathrm{Nil}(R)$, the set of all zero-divisors, the set of all unit elements of $R$, the set of all maximal ideals of $R$ and the set of all nilpotent elements of $R$, respectively. For a subset $A$ of a $\operatorname{ring} R$, we let $A^{*}=A \backslash\{0\}$. The ring $R$ is said to be reduced if it has no non-zero nilpotent element. Some more definitions about commutative rings can be find in $[3,5,15]$.

We use the standard terminology of graphs following [7,14]. Let $G=(V, E)$ be a graph, where $V=V(G)$ is the set of vertices and $E=E(G)$ is the set of edges. By $\bar{G}$, we mean the complement graph of $G$. We write $u-v$, to denote an edge with ends $u, v$. A graph $H=\left(V_{0}, E_{0}\right)$ is called a subgraph of $G$ if $V_{0} \subseteq V$ and $E_{0} \subseteq E$. Moreover, $H$ is called an induced subgraph by $V_{0}$, denoted by $G\left[V_{0}\right]$, if $V_{0} \subseteq V$ and $E_{0}=\left\{u, v \in E \mid u, v \in V_{0}\right\}$. Also $G$ is called a null graph if it has no edge. A complete graph of $n$ vertices is denoted by $K_{n}$. An $n$-partite graph is one whose vertex set can be partitioned into $n$ subsets, so that no edge has both ends in any one subset. A complete $n$-partite graph is one in which each vertex is jointed to every vertex that is not in the same subset. A clique of $G$ is a maximal complete subgraph of $G$ and the number of vertices in the largest clique of $G$, denoted by $\omega(G)$, is called the clique number of $G$. For a graph $G$, let $\chi(G)$ denote the chromatic number of $G$, i.e., the minimal number of colors which can be assigned to the vertices of $G$ in such a way that every two adjacent vertices have different colors. Note that for every graph $G$, $\omega(G) \leq \chi(G)$. A graph $G$ is said to be weakly perfect if $\omega(G)=\chi(G)$. A perfect graph $G$ is a graph in which the chromatic number of every induced subgraph equals the size of the largest clique of that subgraph.

Using the Strong Perfect Graph Theorem, in Section 2 we completely determine all Artinian rings for which the nilpotent graph of $R$ is perfect, leading to our main theorem. In Section 3 for an Artinian ring $R$, it is shown that the graph $\Gamma_{N}(R)$ is weakly perfect. Moreover, the exact value of the $\chi\left(\Gamma_{N}(R)\right)$ is given.

\section{On Perfect Graph}

We start with some properties of the nilpotent elements of a ring. The following remark is useful in our proofs.

Remark 2.1. ([10, Remark 2, 3]). Let $R$ be a non-reduced ring. Then the following statements hold.

(1) For every $x \in \operatorname{Nil}(R)^{*}, x$ is adjacent to all non-zero elements of $R$ and so $Z_{N}(R)=R$.

(2) $\Gamma_{N}(R)\left[\operatorname{Nil}(R)^{*}\right]$ is a (induced) complete subgraph of $\Gamma_{N}(R)$.

To prove our main results we need the following celebrate theorem. 
Theorem 2.1 (The Strong Perfect Graph Theorem [7]). A graph $G$ is perfect if and only if neither $G$ nor $\bar{G}$ contains an induced odd cycle of length at least 5 .

The following result, which is proved in [1, Corollary 2.2], will be helpful in our main results and used frequently in the sequel.

Corollary 2.1. Let $G$ be a graph and $\left\{V_{1}, V_{2}\right\}$ be a partition of $V(G)$. If $G\left[V_{i}\right]$ is a complete graph, for every $1 \leq i \leq 2$, then $G$ is a perfect graph.

The following lemmas have a key role in this paper.

Lemma 2.1. Let $n$ be a positive integer and $R \cong R_{1} \times R_{2} \times \cdots \times R_{n}$, where $R_{i}$ is a ring, for every $1 \leq i \leq n$. If $\Gamma_{N}(R)$ contains no induced odd cycle of length at least 5 , then $n \leq 4$.

Proof. Suppose that $n \geq 5$. Then we can easily get

$$
\begin{aligned}
& (1,0,0,1,0,0, \ldots, 0)-(0,1,0,0,1,0, \ldots, 0)-(1,0,1,0,0,0, \ldots, 0) \\
& -(0,0,0,1,1,0, \ldots, 0)-(0,1,1,0,0,0, \ldots, 0)-(1,0,0,1,0,0, \ldots, 0)
\end{aligned}
$$

is a cycle of length 5. Thus, Theorem 2.1 lead to a contradiction. So, $n \leq 4$.

Before proving first main result of this paper, we bring the following remark, which shows that Artinian rings share the following nice property.

Remark 2.2. Let $R \cong R_{1} \times \cdots \times R_{n}, a=\left(x_{1}, x_{2}, \ldots, x_{n}\right)$ and $b=\left(y_{1}, y_{2}, \ldots, y_{n}\right)$, where $n$ is a positive integer, every $R_{i}$ is an Artinian local ring and $x_{i}, y_{i} \in R_{i}$ for every $1 \leq i \leq n$. Then

(1) $a$ is adjacent to $b$ in $\Gamma_{N}(R)$ if and only if $x_{i} y_{i} \in \operatorname{Nil}\left(R_{i}\right)$ for all $1 \leq i \leq n$;

(2) $a$ is not adjacent to $b$ in $\Gamma_{N}(R)$ if and only if $x_{j} y_{j} \in \mathrm{U}\left(R_{j}\right)$ for some $1 \leq j \leq n$;

(3) $a$ is adjacent to $b$ in $\overline{\Gamma_{N}(R)}$ if and only if $x_{i} y_{i} \in \mathrm{U}\left(R_{i}\right)$ for some $1 \leq i \leq n$;

(4) $a$ is not adjacent to $b$ in $\overline{\Gamma_{N}(R)}$ if and only if $x_{j} y_{j} \in \operatorname{Nil}\left(R_{j}\right)$ for all $1 \leq j \leq n$.

By using a similar way as used in the proof of [1, Lemma 2.3], one can prove the following result.

Lemma 2.2. Let $S_{1}, S_{2}, S_{3}, S_{4}$ be rings such that $S_{1} \cong R_{1}, S_{2} \cong R_{1} \times R_{2}, S_{3} \cong$ $R_{1} \times R_{2} \times R_{3}$ and $S_{4} \cong R_{1} \times R_{2} \times R_{3} \times R_{4}$, where $R_{i}$ is a ring for every $1 \leq i \leq 4$. Then, if $\Gamma_{N}\left(S_{4}\right)$ is a perfect graph, then $\Gamma_{N}\left(S_{3}\right), \Gamma_{N}\left(S_{2}\right)$ and $\Gamma_{N}\left(S_{1}\right)$ are perfect graphs.

We are now in a position to state our first main result in this section.

Theorem 2.2. Let $R$ be a non-reduced Artinian ring. Then $\Gamma_{N}(R)$ is a perfect graph if and only if $|\operatorname{Max}(\mathrm{R})| \leq 4$.

Proof. For one direction assume that $|\operatorname{Max}(\mathrm{R})| \leq 4$. This together with $[3$, Theorem 8.7] implies that there exists a positive integer $n$ such that $R \cong R_{1} \times \cdots \times R_{n}$, where $R_{i}$ is an Artinian local ring, for every $1 \leq i \leq n$ and $n \leq 4$. By Theorem 2.1, it is enough to show that $\Gamma_{N}(R)$ and $\overline{\Gamma_{N}(R)}$ contains no induced odd cycle of length at 
least 5. By Lemma 2.2, we need to prove the case $n=4$. So let $R \cong R_{1} \times R_{2} \times R_{3} \times R_{4}$, where $R_{i}$ is an Artinian local ring. We have the following two claims.

Claim 1. $\Gamma_{N}(R)$ contains no induced odd cycle of length at least 5. Note that if $R$ is an Artinian non-reduced ring, then $Z_{N}(R)=R=U(R) \cup Z(R)$, where $U(R)=U\left(R_{1}\right) \times \cdots \times U\left(R_{4}\right)$. We consider the following partition for non-zero zero-divisors of $R$ :

$$
\begin{aligned}
& A=\left\{\left\{\left(x_{1}, x_{2}, x_{3}, x_{4}\right) \mid x_{i} \in \operatorname{Nil}\left(R_{i}\right) \text { for all } i\right\} \backslash\{(0,0,0,0)\}\right\} \\
& B=\left\{\left(x_{1}, x_{2}, x_{3}, x_{4}\right) \mid \text { for some } i, x_{i} \notin \operatorname{Nil}\left(R_{i}\right)\right\}
\end{aligned}
$$

Thus $A \cap B=\varnothing, A \cap U(R)=\varnothing, B \cap U(R)=\varnothing$ and $V\left(\Gamma_{N}(R)\right)=A \cup B \cup U(R)$. Also we consider the following partition for $B$ :

$$
\begin{aligned}
& B_{1}=\left\{(x, y, z, w) \in B \mid x \in \mathrm{U}\left(R_{1}\right)\right\} \\
& B_{2}=\left\{(x, y, z, w) \in B \mid x \in \operatorname{Nil}\left(R_{1}\right) \text { and } y \in \mathrm{U}\left(R_{2}\right)\right\} \\
& B_{3}=\left\{(x, y, z, w) \in B \mid x \in \operatorname{Nil}\left(R_{1}\right), y \in \operatorname{Nil}\left(R_{2}\right) \text { and } z \in \mathrm{U}\left(R_{3}\right)\right\} \\
& B_{4}=\left\{(x, y, z, w) \in B \mid x \in \operatorname{Nil}\left(R_{1}\right), y \in \operatorname{Nil}\left(R_{2}\right), z \in \operatorname{Nil}\left(R_{3}\right) \text { and } w \in \mathrm{U}\left(R_{4}\right)\right\} .
\end{aligned}
$$

It is easy to see that $B=\cup_{i=1}^{4} B_{i}$ and $B_{i} \cap B_{j}=\varnothing$ for every $i \neq j$. The elements of $V\left(\Gamma_{N}(R)\right)$ have form $a_{i}=\left(x_{i}, y_{i}, z_{i}, w_{i}\right)$, where $x_{i} \in R_{1}, y_{i} \in R_{2}, z_{i} \in R_{3}$ and $w_{i} \in R_{4}$ for each $i \in \mathbb{N}$. Now, assume to the contrary that $a_{1}-a_{2}-\cdots-a_{n}-a_{1}$ is an induced odd cycle of length at least 5 in $\Gamma_{N}(R)$. We have the following cases.

Case 1. $\left\{a_{1}, \ldots, a_{n}\right\} \cap \mathrm{U}(R)=\varnothing$. Assume to the contrary and with no loss of generality that $a_{1}=\left(x_{1}, y_{1}, z_{1}, w_{1}\right) \in \mathrm{U}(R)$. Then $a_{2}$ and $a_{n}$ must be in $\operatorname{Nil}(R)^{*}$. Therefore, $a_{n}$ is adjacent to $a_{2}$, which is a contradiction.

Case 2. $\left\{a_{1}, \ldots, a_{n}\right\} \cap A=\varnothing$. Let $a_{i} \in\left\{a_{1}, \ldots, a_{n}\right\} \cap A$, for some $1 \leq i \leq n$. Then by Remark 2.1, $a_{i}$ is adjacent to all other vertices, a contradiction. Thus, $\left\{a_{1}, \ldots, a_{n}\right\} \cap A=\varnothing$.

Case 3. $\left\{a_{1}, \ldots, a_{n}\right\} \cap B_{4}=\varnothing$. To show this, for a contradiction assume that $a_{1}=\left(x_{1}, y_{1}, z_{1}, w_{1}\right) \in B_{4}$. Since $a_{2}$ and $a_{n}$ are adjacent to $a_{1}$ and

$$
a_{1} \in \operatorname{Nil}\left(R_{1}\right) \times \operatorname{Nil}\left(R_{2}\right) \times \operatorname{Nil}\left(R_{3}\right) \times \mathrm{U}\left(R_{4}\right),
$$

we see that the fourth components of $a_{2}$ and $a_{n}$ must be in $\operatorname{Nil}\left(R_{4}\right)$. Now since $x_{3} x_{1}, y_{1} y_{3}$ and $z_{1} z_{3}$ are nilpotent elements and $a_{3}$ is not adjacent to $a_{1}$, by Part (2) of Remark 2.2, we conclude that the fourth component of $a_{3}$ must be in $\mathrm{U}\left(R_{4}\right)$. This together with the fact that $a_{4}$ is adjacent to $a_{3}$ imply that the fourth component of $a_{4}$ is nilpotent element and so $a_{4} a_{1} \in \operatorname{Nil}(R)$. Therefore, $a_{4}$ is adjacent to $a_{1}$, which is a contradiction. So the assertion is proved.

Case 4. $\left\{a_{1}, \ldots, a_{n}\right\} \cap B_{1}=\varnothing$. Assume to the contrary and with no loss of generality, $a_{1}=\left(x_{1}, y_{1}, z_{1}, w_{1}\right) \in B_{1}$. It is easy to see that for every $1 \leq i \leq 4$, there is no edge between any two vertices of $B_{i}$. This together with the above cases imply that $a_{n}$ and $a_{2}$ are in $B_{2} \cup B_{3}$. We distinguish the following three subcases. 
Subcase 4.1. $\left\{a_{n}, a_{2}\right\} \subset B_{3}$. In this case, we have

$$
\left\{a_{n}, a_{2}\right\} \subset \operatorname{Nil}\left(R_{1}\right) \times \operatorname{Nil}\left(R_{2}\right) \times \mathrm{U}\left(R_{3}\right) \times R_{4} .
$$

Then the third components of $a_{1}$ and $a_{3}$ must be in $\operatorname{Nil}\left(R_{3}\right)$. Also, since $a_{n}$ is not adjacent to $a_{3}$, by Part (2) of Remark 2.2, the fourth components of $a_{n}$ and $a_{3}$ must be in $\mathrm{U}\left(R_{4}\right)$. This yields

$$
\begin{aligned}
& a_{1} \in \mathrm{U}\left(R_{1}\right) \times R_{2} \times \operatorname{Nil}\left(R_{3}\right) \times R_{4}, \\
& a_{3} \in R_{1} \times R_{2} \times \operatorname{Nil}\left(R_{3}\right) \times \mathrm{U}\left(R_{4}\right), \\
& a_{n} \in \operatorname{Nil}\left(R_{1}\right) \times \operatorname{Nil}\left(R_{2}\right) \times \mathrm{U}\left(R_{3}\right) \times \mathrm{U}\left(R_{4}\right) .
\end{aligned}
$$

Then the fourth components of $a_{1}$ and $a_{2}$ must be in $\operatorname{Nil}\left(R_{4}\right)$. Hence, we find that

$$
\begin{aligned}
& a_{1} \in \mathrm{U}\left(R_{1}\right) \times R_{2} \times \operatorname{Nil}\left(R_{3}\right) \times \operatorname{Nil}\left(R_{4}\right), \\
& a_{2} \in \operatorname{Nil}\left(R_{1}\right) \times \operatorname{Nil}\left(R_{2}\right) \times \mathrm{U}\left(R_{3}\right) \times \operatorname{Nil}\left(R_{4}\right) .
\end{aligned}
$$

Now, since $a_{2}$ is not adjacent to $a_{4}$, the third components of $a_{4}$ must be in $\mathrm{U}\left(R_{3}\right)$. This implies that $a_{4}$ is not adjacent to $a_{n}$ and so $n \geq 7$. It is easy to see that the third component of $a_{5}$ must be in $\operatorname{Nil}\left(R_{3}\right)$ and so $a_{5} a_{2} \in \operatorname{Nil}(R)$. This implies that $a_{5}-a_{2}$, a contradiction. So, in this case the assertion is proved.

Subcase 4.2. $\left\{a_{n}, a_{2}\right\} \subset B_{2}$. By a similar way as used in Subcase (4.1), we get a contradiction.

Subcase 4.3. $a_{n} \in B_{2}$ and $a_{2} \in B_{3}$. By a similar way as used in Subcase (4.1), we get a contradiction. Thus $\left\{a_{1}, \ldots, a_{n}\right\} \cap B_{1}=\varnothing$.

By the above cases, $\left\{a_{1}, \ldots, a_{n}\right\} \subseteq B_{2} \cup B_{3}$, but this is contradicts the fact $\Gamma_{N}(R)\left[B_{2} \cup B_{3}\right]$ is a bipartite graph, and thus, $\Gamma_{N}(R)$ contains no induced odd cycle of length at least 5 .

In Claim 2, $\mathrm{U}(R), A, B$ and $B_{i}$ are sets that mentioned in Claim 1.

Claim 2. $\overline{\Gamma_{N}(R)}$ contains no induced odd cycle of length at least 5 . We show that $\overline{\Gamma_{N}(R)}$ contains no induced odd cycle at least 5. Assume to the contrary that

$$
a_{1}-a_{2}-\cdots-a_{n}-a_{1}
$$

is an induced odd cycle of length at least 5 in $\overline{\Gamma_{N}(R)}$. It is clear that $\overline{\Gamma_{N}(R)}[A]$ is a null graph and so $\left\{a_{1}, \ldots, a_{n}\right\} \cap A=\varnothing$. Also, we show that

$$
\left\{a_{1}, \ldots, a_{n}\right\} \cap \mathrm{U}(R)=\varnothing .
$$

Assume to the contrary and with no loss of generality that $a_{1} \in \mathrm{U}(R)$. Obviously, $a_{1}$ is just adjacent to all of vertices of $Z_{N}(R) \backslash \mathrm{Nil}(R)$. This together with the fact that $\left\{a_{1}, \ldots, a_{n}\right\} \subset Z_{N}(R) \backslash \operatorname{Nil}(R)$ imply that $a_{1}$ is adjacent to all other vertices, a contradiction. Thus $\left\{a_{1}, \ldots, a_{n}\right\} \cap \mathrm{U}(R)=\varnothing$. We claim that

$$
\left\{a_{1}, \ldots, a_{n}\right\} \cap B_{4}=\varnothing .
$$

Indeed, if not, there would exist an $a_{i} \in B_{4}$. Without loss of generality, we may assume that $a_{1}=\left(x_{1}, y_{1}, z_{1}, w_{1}\right) \in B_{4}$. Then $a_{1} \in \operatorname{Nil}\left(R_{1}\right) \times \operatorname{Nil}\left(R_{2}\right) \times \operatorname{Nil}\left(R_{3}\right) \times \mathrm{U}\left(R_{4}\right)$. This 
together with Part (3) of Remark 2.2 implies that the forth components of $a_{2}$ and $a_{n}$ must be in $\mathrm{U}\left(R_{4}\right)$ and so we have

$$
\begin{aligned}
& a_{n} \in R_{1} \times R_{2} \times R_{3} \times \mathrm{U}\left(R_{4}\right), \\
& a_{2} \in R_{1} \times R_{2} \times R_{3} \times \mathrm{U}\left(R_{4}\right) .
\end{aligned}
$$

It is easy to see that $a_{2}$ is adjacent to $a_{n}$, a contradiction, and so,

$$
\left\{a_{1}, \ldots, a_{n}\right\} \cap B_{4}=\varnothing .
$$

Finally to complete the proof, we prove that $\left\{a_{1}, \ldots, a_{n}\right\} \cap B_{3}=\varnothing$. To get a contradiction, let $a_{1}=\left(x_{1}, y_{1}, z_{1}, w_{1}\right) \in B_{3}$. Then

$$
a_{1} \in \operatorname{Nil}\left(R_{1}\right) \times \operatorname{Nil}\left(R_{2}\right) \times \mathrm{U}\left(R_{3}\right) \times R_{4} .
$$

Since $a_{1}-a_{n}, a_{1}-a_{2}$ and $a_{2}$ is not adjacent to $a_{n}$, we consider the following two cases.

Case 1.

$$
\begin{aligned}
& a_{1} \in \operatorname{Nil}\left(R_{1}\right) \times \operatorname{Nil}\left(R_{2}\right) \times \mathrm{U}\left(R_{3}\right) \times \mathrm{U}\left(R_{4}\right), \\
& a_{2} \in R_{1} \times R_{2} \times \mathrm{U}\left(R_{3}\right) \times \operatorname{Nil}\left(R_{4}\right), \\
& a_{n} \in R_{1} \times R_{2} \times \operatorname{Nil}\left(R_{3}\right) \times \mathrm{U}\left(R_{4}\right) .
\end{aligned}
$$

Since $a_{3}$ is not adjacent to $a_{1}$, the third and the fourth components $a_{3}$ must be nilpotent. On the other hand, $a_{3}$ is adjacent to $a_{2}$. This implies that $x_{3} x_{2} \in \mathrm{U}\left(R_{1}\right)$ or $y_{2} y_{3} \in \mathrm{U}\left(R_{2}\right)$.

First suppose that $x_{3} x_{2} \in \mathrm{U}\left(R_{1}\right)$. Now, we know that

$$
\begin{aligned}
& a_{3} \in \mathrm{U}\left(R_{1}\right) \times R_{2} \times \operatorname{Nil}\left(R_{3}\right) \times \operatorname{Nil}\left(R_{4}\right), \\
& a_{2} \in \mathrm{U}\left(R_{1}\right) \times R_{2} \times \mathrm{U}\left(R_{3}\right) \times \operatorname{Nil}\left(R_{4}\right) .
\end{aligned}
$$

This together with that $a_{3}$ is adjacent to $a_{4}$ implies that $x_{3} x_{4} \in \mathrm{U}\left(R_{1}\right)$ or $y_{3} y_{4} \in \mathrm{U}\left(R_{2}\right)$. If $x_{3} x_{4} \in \mathrm{U}\left(R_{1}\right)$, then we have $x_{2} x_{4} \in \mathrm{U}\left(R_{1}\right)$. Therefore, $a_{4}$ is adjacent to $a_{2}$, which is a contradiction. Thus, we conclude that $y_{3} y_{4} \in \mathrm{U}\left(R_{2}\right)$. This yields

$$
\begin{aligned}
& a_{3} \in \mathrm{U}\left(R_{1}\right) \times \mathrm{U}\left(R_{2}\right) \times \operatorname{Nil}\left(R_{3}\right) \times \operatorname{Nil}\left(R_{4}\right), \\
& a_{4} \in \operatorname{Nil}\left(R_{1}\right) \times \mathrm{U}\left(R_{2}\right) \times R_{3} \times R_{4} .
\end{aligned}
$$

Since $a_{4}$ is not adjacent to $a_{1}$, we have

$$
a_{4} \in \operatorname{Nil}\left(R_{1}\right) \times \mathrm{U}\left(R_{2}\right) \times \operatorname{Nil}\left(R_{3}\right) \times \operatorname{Nil}\left(R_{4}\right) .
$$

Thus $a_{4}$ is not adjacent to $a_{n}$ and so $n \geq 7$. On the other hand, since $a_{4}-a_{5}$, the second components of $a_{5}$ must be unit and so $a_{5}$ is adjacent to $a_{2}$, which is a contradiction.

So, suppose that $y_{2} y_{3} \in \mathrm{U}\left(R_{2}\right)$. Similarly, we get a contradiction. Thus in this case the assertion is proved. 


\section{Case 2.}

$$
\begin{aligned}
& a_{1} \in \operatorname{Nil}\left(R_{1}\right) \times \operatorname{Nil}\left(R_{2}\right) \times \mathrm{U}\left(R_{3}\right) \times \mathrm{U}\left(R_{4}\right), \\
& a_{2} \in R_{1} \times R_{2} \times \operatorname{Nil}\left(R_{3}\right) \times \mathrm{U}\left(R_{4}\right), \\
& a_{n} \in R_{1} \times R_{2} \times \mathrm{U}\left(R_{3}\right) \times \operatorname{Nil}\left(R_{4}\right) .
\end{aligned}
$$

By similar argument that of Case 1 , we get a contradiction.

This means that $\left\{a_{1}, \ldots, a_{n}\right\} \subseteq B_{2} \cup B_{1}$. Clearly, $\overline{\Gamma_{N}(R)}\left[B_{1}\right], \overline{\Gamma_{N}(R)}\left[B_{2}\right]$ are complete, and thus by Corollary 2.1, $\overline{\Gamma_{N}(R)}\left[B_{1} \cup B_{2}\right]$ is a perfect graph, a contradiction. Hence $\overline{\Gamma_{N}(R)}$ contain no induced odd cycle of length at least 5 . Therefore, by Claim 1, Claim 2 and Theorem 2.1, $\Gamma_{N}(R)$ is a perfect graph.

For the other direction, since $R \cong R_{1} \times \cdots \times R_{n}$, where $R_{i}$ is an Artinian local ring, for every $1 \leq i \leq n$, then by Theorem 2.1 and Lemma 2.1, $n \leq 4$, as desired.

\section{The Nilpotent Graph of an Artinian Ring is Weakly Perfect}

The main goal of this section is to study the coloring of the nilpotent graphs of Artinian rings. For an Artinian ring $R$, it is shown that the graph $\Gamma_{N}(R)$ is weakly perfect. Moreover, the exact value of the $\chi\left(\Gamma_{N}(R)\right)$ is given.

Theorem 3.1. Let $R$ be an Artinian ring. Then

$$
\omega\left(\Gamma_{N}(R)\right)=\chi\left(\Gamma_{N}(R)\right)=\left|\operatorname{Nil}(R)^{*}\right|+|\operatorname{Max}(R)| .
$$

Proof. First let $R$ be an Artinian local ring. One may easily check that $V\left(\Gamma_{N}(R)\right)=$ $\operatorname{Nil}(R) \cup \mathrm{U}(R)$ and so $\{\operatorname{Nil}(R), \mathrm{U}(R)\}$ is a partition of $V\left(\Gamma_{N}(R)\right)$. By Remark 2.1, we have $\Gamma_{N}(R)\left[\operatorname{Nil}(R)^{*}\right]$ is a complete subgraph of $\Gamma_{N}(R)$ and every vertex $x \in \operatorname{Nil}(R)^{*}$ is adjacent to all other vertices. This together with this fact that there is no adjacency between two vertices of $\mathrm{U}(R)$ imply that $\Gamma_{N}(R)=\Gamma_{N}(R)\left[\operatorname{Nil}(R)^{*}\right] \vee \Gamma_{N}(R)[\mathrm{U}(R)]$ and so

$$
\omega\left(\Gamma_{N}(R)\right)=\chi\left(\Gamma_{N}(R)\right)=\omega\left(\Gamma_{N}(R)\left[\operatorname{Nil}(R)^{*}\right]\right)+\omega\left(\Gamma_{N}(R)[\mathrm{U}(R)]\right)=\left|\operatorname{Nil}(R)^{*}\right|+1 .
$$

Now, let $R$ be an Artinian non-local ring. By [3, Theorem 8.7], one can deduce that there exists a positive integer $n$ such that $R \cong R_{1} \times \cdots \times R_{n}$, where $R_{i}$ is an Artinian local ring, for every $1 \leq i \leq n$. We put:

$$
\begin{aligned}
A & =\left\{\left\{\left(x_{1}, \ldots, x_{n}\right) \mid x_{i} \in \operatorname{Nil}\left(R_{i}\right) \text { for all } 1 \leq i \leq n\right\} \backslash\{(0,0,0,0)\}\right\}, \\
B & =\left\{\left(x_{1}, \ldots, x_{n}\right) \mid \text { for some } i, x_{i} \notin \operatorname{Nil}\left(R_{i}\right)\right\} \\
\mathrm{U}(R) & =\left\{\left(x_{1}, \ldots, x_{n}\right) \mid x_{i} \in \mathrm{U}\left(R_{i}\right) \text { for all } 1 \leq i \leq n\right\} .
\end{aligned}
$$

One may easily check that $V\left(\Gamma_{N}(R)\right)=A \cup B \cup \mathrm{U}(R), A \cap B=\varnothing, A \cap \mathrm{U}(R)=$ $\varnothing, B \cap \mathrm{U}(R)=\varnothing$ and so $\{A, B, \mathrm{U}(R)\}$ is a partition of $V\left(\Gamma_{N}(R)\right)$. It is clear that $\Gamma_{N}(R)[U(R)]=\overline{K_{|\mathrm{U}(R)|}}$ and there is no adjacency between two vertices of $B$ and $\mathrm{U}(R)$. To complete the proof, we prove that

$$
\begin{aligned}
\Gamma_{N}(R)[A \cup B] & =\Gamma_{N}(R)[A] \vee \Gamma_{N}(R)[B], \\
\Gamma_{N}(R)[A \cup \mathrm{U}(R)] & =\Gamma_{N}(R)[A] \vee \Gamma_{N}(R)[\mathrm{U}(R)],
\end{aligned}
$$


where $\Gamma_{N}(R)[A]$ is a complete subgraph of $\Gamma_{N}(R)$ and $\Gamma_{N}(R)[B]$ is an $n$-partite subgraph of $\Gamma_{N}(R)$, which is not an $(n-1)$-partite subgraph of $\Gamma_{N}(R)$. To see this, by Part (1) of Remark 2.1, we have $\Gamma_{N}(R)[A]$ is a complete subgraph of $\Gamma_{N}(R)$ and every vertex $x \in A$ is adjacent to all other vertices.

Now, for every $1 \leq i \leq n$, let $B_{i}=\left\{\left(x_{1}, \ldots, x_{n}\right) \in B \mid x_{i} \in \mathrm{U}\left(R_{i}\right)\right.$ and $x_{j} \in \operatorname{Nil}\left(R_{j}\right)$ for every $1 \leq j \leq i$ \}. It is easy to see that for every $1 \leq i \leq n$, there is no adjacency between two vertices of $B_{i}$. This together with this fact that the set $\{(1,0,0, \ldots, 0),(0,1,0, \ldots, 0), \ldots,(0,0,0, \ldots, 1)\}$ is a clique of $\Gamma_{N}(R)[B]$ imply that $\Gamma_{N}(R)[B]$ is an $n$-partite subgraph of $\Gamma_{N}(R)$, which is not an $(n-1)$-partite subgraph of $\Gamma_{N}(R)$. Therefore,

$$
\begin{aligned}
\Gamma_{N}(R)[A \cup B] & =\Gamma_{N}(R)[A] \vee \Gamma_{N}(R)[B], \\
\Gamma_{N}(R)[A \cup \mathrm{U}(R)] & =\Gamma_{N}(R)[A] \vee \Gamma_{N}(R)[\mathrm{U}(R)]
\end{aligned}
$$

and so

$$
\omega\left(\Gamma_{N}(R)\right)=\chi\left(\Gamma_{N}(R)\right)=\omega\left(\Gamma_{N}(R)[A]\right)+\omega\left(\Gamma_{N}(R)[B]\right)=\left|\operatorname{Nil}(R)^{*}\right|+|\operatorname{Max}(R)|
$$

and the proof is complete.

We close this paper with the following result.

Theorem 3.2. Let $R$ be a non-reduced ring. Then the following statements are equivalent:

(1) $\omega\left(\Gamma_{N}(R)\right)=2$;

(2) $\chi\left(\Gamma_{N}(R)\right)=2$;

(3) either $\Gamma_{N}(R) \cong K_{1,2}$ or $\Gamma_{N}(R) \cong K_{1} \vee \overline{K_{\infty}}$.

Proof. (3) $\Rightarrow(1),(2)$ are clear. (2) $\Rightarrow(3)$ is obtained by similar argument to that proof of $(1) \Rightarrow(3)$. (1) $\Rightarrow(3)$ is only thing to prove.

$(1) \Rightarrow(3)$. Suppose that $\omega\left(\Gamma_{N}(R)\right)=2$. First we show that $\left|\operatorname{Nil}(R)^{*}\right|=1$. To see this, consider $A=\{a, b, c\}$ where $a, b \in \mathrm{Nil}(R)^{*}$ and $c \in \mathrm{U}(R)$. Then the subgraph induced by $A$ is isomorphic to $K_{3}$, a contradiction. Thus, $\left|\operatorname{Nil}(R)^{*}\right|=1$.

Now, we have two following cases.

Case 1. $Z(R)=\operatorname{Nil}(R)$. Since $\left|Z(R)^{*}\right|=1<\infty, R$ is an Artinian (indeed $R$ is finite). By [3, Theorem 8.7] there exists a positive integer $n$ such that $R \cong R_{1} \times \cdots \times R_{n}$, where each $R_{i}, 1 \leq i \leq n$, is an Artinian local ring. If $n \geq 2$, then $Z(R)^{*} \geq 2$, a contradiction. So we may assume that $R$ is an Artinian local ring. This, together $[8$, Example 1.5], implies that $R \cong \mathbb{Z}_{4}$ or $\frac{\mathbb{Z}_{2}[x]}{\left\langle x^{2}\right\rangle}$ and so $\Gamma_{N}(R) \cong K_{1,2}$.

Case 2. $Z(R) \neq \operatorname{Nil}(R)$. Since $\omega\left(\Gamma_{N}(R)\right)=2$ and by Remark 2.1, every $x \in$ $\operatorname{Nil}(R)^{*}, x$ is adjacent to all non-zero elements of $R$, we have only to show that $|Z(R)|=\infty$. To get a contradiction, let $|Z(R)|<\infty$. Then by [3, Theorem 8.7], we may write $R \cong R_{1} \times \cdots \times R_{n}$, where $R_{i}$ is an Artinian local ring, for every $1 \leq i \leq n$. Since $Z(R) \neq \operatorname{Nil}(R)$, we have $n \geq 2$. Also, since $R$ is non-reduced, without loss of generality, we can suppose that $a \in \operatorname{Nil}\left(R_{1}\right)^{*}$. Consider $\phi=\{x, y, z\}$, where $x=(a, 0, \ldots, 0), y=(1,0, \ldots, 0), z=(0,1,0, \ldots, 0)$. Then the subgraph induced 
by $\phi$ in $\Gamma_{N}(R)$ is isomorphic to $K_{3}$, a contradiction. Thus, $|Z(R)|=\infty$ and so $\Gamma_{N}(R) \cong K_{1} \vee \overline{K_{\infty}}$ and the proof is complete.

Acknowledgements. The authors thank to the referees for their careful reading and their excellent suggestions.

\section{REFERENCES}

[1] V. Aghapouramin and M. J. Nikmehr, On perfectness of a graph associated with annihilating ideals of a ring, Discrete Math. Algorithms Appl. 10(04) (2018), 201-212 .

[2] D. F. Anderson and P. S. Livingston, The zero-divisor graph of a commutative ring, J. Algebra 217 (1999), 434-447.

[3] M. F. Atiyah and I. G. Macdonald, Introduction to Commutative Algebra, Addison-Wesley Publishing Company, Massachusetts, London, Ontario,1969.

[4] I. Beck, Coloring of commutative rings, J. Algebra 116 (1988), 208-226.

[5] W. Bruns and J. Herzog, Cohen-Macaulay Rings, Cambridge University Press, Cambridge, 1997.

[6] P. W. Chen, A kind of graph structure of rings, Algebra Colloq. 10(2) (2003), 229-238.

[7] R. Diestel, Graph Theory, Springer-Verlag, New York, USA, 2000.

[8] R. Kala and S. Kavitha, Nilpotent graphs of genus one, Discrete Math. Algorithms Appl. 6 (2014), $1450-1463$.

[9] A. Li and Q. Li, A kind of graph of structure on von-Neumann regular rings, Int. J. Algebra 4(6) (2010), 291-302.

[10] A. H. Li and Q. H. Li, A kind of graph structure on non-reduced rings, Algebra Colloq. 17(1) (2010), 173-180.

[11] M. J. Nikmehr, R. Nikandish and M. Bakhtyiari, On the essential graph of a commutative ring, J. Algebra Appl. (2017), 175-189.

[12] A. Patil, B. N. Waphare and V. Joshi, Perfect zero-divisor graphs, Discrete Math. 340 (2017), $740-745$.

[13] B. Smith, Perfect zero-divisor graphs of $\mathbb{Z}_{n}$, Rose-Hulman Undergrad. Math J. 17 (2016), $114-132$.

[14] D. B. West, Introduction to Graph Theory, 2nd Edition, Prentice Hall, Upper Saddle River, 2001.

[15] R. Wisbauer, Foundations of Module and Ring Theory, Breach Science Publishers, Reading, 1991.

${ }^{1}$ Faculty of Mathematics,

K. N. Toosi University of Technology,

TEHRAN, Iran

Email address: nikmehr@kntu.ac.ir

Email address: abdoreza.azadi@email.kntu.ac.ir 